\title{
Cortical and Internal Watershed Infarcts Might Be Key Signs for Predicting Neurological Deterioration in Patients with Internal Carotid Artery Occlusion with Mild Symptoms
}

\author{
Yuki Amano $^{a}$ Hiroyasu Sano $^{b}$ Ayataka Fujimoto $^{c}$ Hiroaki Kenmochi $^{a}$ \\ Haruhiko Sato $^{a}$ Soichi Akamine ${ }^{b}$ \\ a Department of Neurosurgery, Seirei Mikatahara General Hospital, Hamamatsu, Japan; \\ ${ }^{b}$ Department of Stroke Center, Seirei Mikatahara General Hospital, Seirei Hamamatsu \\ General Hospital, Hamamatsu, Japan; ' Epilepsy Center, Seirei Hamamatsu General Hospital, \\ Hamamatsu, Japan
}

\begin{abstract}
Keywords
Large vessel occlusion - Watershed infarction - Mild symptom - Lenticulostriate arteries .

Internal carotid artery occlusion · Cardioembolism
\end{abstract}

\begin{abstract}
Background: Treatment for acute ischemic stroke due to large vessel occlusion (LVO) with mild symptoms is under discussion. Although most patients have good outcomes, some patients deteriorate and have unfavorable results. Imaging findings that predict the prognosis of LVO with mild symptoms are needed to identify patients who require treatment. In this study, we focused on watershed infarctions (WSIs), because this clinical phenomenon quite sensitively reflects changes in cerebral blood flow. The purpose of this study was to assess positive rates of WSI on MRI findings in patients with internal carotid artery (ICA) occlusion, and compare WSI-positive rates between patients divided according to their clinical course. Methods: We retrospectively collected data of 1,531 patients who presented with acute ischemic stroke between June 2006 and July 2019. Among them, we chose symptomatic ICA occlusion patients with a past history of atrial fibrillation who were treated conservatively. We divided these patients into two groups, those with maintenance or improvement in their NIHSS score after hospitalization, and those whose NIHSS score worsened. We compared WSI-positive rates between these two groups. Results: Thirty-seven of the 1,531 patients were included in this study. Of them, total NIHSS score was maintained or improved in 8 patients (group A), 3 of whom (37.5\%) had internal watershed infarctions (IWIs). In group B, consisting of patients whose NIHSS score worsened by $>2$ at 7 days from symptom onset, 24 (82.8\%)
\end{abstract}


had IWIs. Group A thus had statistically lower IWI positivity rates than group B ( $p=0.02)$. Three patients (37.5\%) in group A had cortical watershed infarctions (CWIs), while 27 patients in group B (93.1\%) had CWIs. Group A thus had a significantly lower CWI positivity rate than group B ( $p=0.002)$. Conclusion: In patients with mildly symptomatic ICA occlusion, CWIs and IWIs might be key signs for predicting neurological deterioration after hospitalization.

(C) 2020 The Author(s)

Published by S. Karger AG, Basel

\section{Introduction}

Since the publication of several randomized control trials, endovascular thrombectomy has become standard therapy for patients with moderate to severe ischemic stroke and large vessel occlusion (LVO) [1-9]. Based on these trials, current guidelines endorse endovascular thrombectomy as the standard of care for patients with LVO in the anterior circulation (internal carotid artery [ICA] and middle cerebral artery [MCA]) with National Institutes of Health Stroke Scale (NIHSS) scores of 6 or more [10]. This NIHSS cutoff was established because the eligibility criteria of previous RCTs mostly excluded patients with NIHSS scores $<6$.

The endovascular approach for low NIHSS score emergency LVO has been previously reported; however, data are scarce [11,12], and endovascular intervention in such cases remains to be established. Therefore, treatment of patients who present with mild neurological symptoms and are also found to have an intracranial LVO pose an exceptionally difficult therapeutic dilemma. In these cases, the clinical outcome is generally poor [13-15].

Acute ischemic stroke (AIS) with ICA occlusion is usually caused by compromised cerebral perfusion, an embolic event, or a combination of hypoperfusion and embolism [16,17]. Since watershed infarctions (WSIs), which occur at characteristic locations at the junction between two main arterial territories, correlate with both hypoperfusion and microembolism, we focused on WSIs in this study [16, 18-29]. Based on these reports, we hypothesized that patients with ICA occlusion with mild symptoms, and whose initial MRI shows fewer WSIs, are likely to have a good clinical course. The purpose of this study was to compare WSI positivity rates in patients with ICA occlusion with mild symptoms between patients whose NIHSS score remained or improved after hospitalization and those whose NIHSS score worsened. This is the first study to research the correlation between WSIs and mild symptoms due to ICA occlusion.

\section{Methods}

\section{Study Design}

The Ethics Committee of Seirei Mikatahara General Hospital approved the protocol for this study, which was performed in accordance with the principles of the Declaration of Helsinki. Participants in this observational, non-randomized study were identified via a retrospective electronic chart review of AIS patients treated between June 2006 and July 2019 at the Seirei Mikatahara General Hospital.

\section{Patients}

We retrospectively collected the data of 1,531 patients who presented with AIS between June 2006 and July 2019. Patients who met the following criteria were selected from among them: (1) those presenting within $24 \mathrm{~h}$ of symptom onset, (2) those who underwent emer-

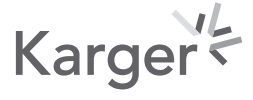




\section{Cerebrovascular Diseases Extra}

\begin{tabular}{l|l}
\hline Cerebrovasc Dis Extra 2020;10:76-83 \\
\hline DOI: 10.1159/000508090 & $\begin{array}{l}\text { @ 2020 The Author(s). Published by S. Karger AG, Basel } \\
\text { www.karger.com/cee }\end{array}$ \\
\hline
\end{tabular}

Amano et al.: Watershed Infarcts Key to Predicting Prognosis in ICA Occlusion

Fig. 1. Classification of watershed infarction: $a$ and $b$, subtypes of cortical watershed infarction; c, subtypes of internal watershed infarction.
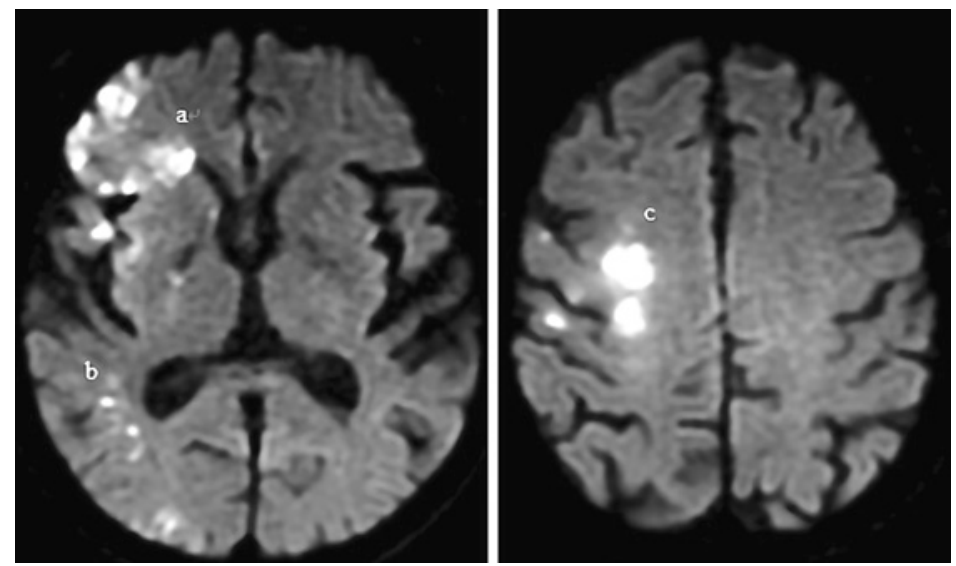

gency brain MRI, including diffusion-weighted imaging (DWI) and magnetic resonance angiography (MRA), (3) who had symptomatic ICA occlusion, and (4) a past history of atrial fibrillation, to extract patients of cardioembolism. ICA occlusion was diagnosed based on the findings of acute MRA. We excluded patients who met the following criteria: (1) those who underwent endovascular treatment, (2) had incomplete early imaging, and (3) early loss to follow-up.

\section{Imaging}

According to our stroke imaging protocol, patients were scheduled to undergo an MRI on admission, and follow-up MRI or computed tomography 5-7 days after symptom onset. The initial and follow-up MRI protocol included DWI, fluid-attenuated inversion recovery (FLAIR), and MRA. Imaging studies were performed using a 1.5-T scanner (Provida) from Philips Healthcare (Best, The Netherlands).

The MR images were analyzed by two experienced stroke neurologists blinded to the clinical data. The patterns of DWI lesions were classified as perforating artery infarctions, pial infarctions, cortical watershed infarctions (CWI), and internal watershed infarctions (IWI). According to classic neuropathology studies [18], CWI was defined as infarctions of the white matter between the cortical territories of the anterior cerebral artery, MCA, and posterior cerebral artery. IWI was defined as infarctions of the white matter between the deep and superficial arterial systems of the MCA, or between the superficial systems of the MCA and anterior cerebral artery (Fig. 1). Arterial occlusion was defined as complete loss of the digital flow signal on MRA. The presence of MCA flow and collateral flow of the anterior communicating artery and posterior communicating artery was defined by signal positivity on MRA.

\section{Clinical and Statistical Analysis}

We assessed NIHSS scores at 7 days from symptom onset and divided patients into two groups. Patients who satisfied the following criteria were categorized as group A: (1) NIHSS score remained unchanged or improved at 7 days from symptom onset, (2) NIHSS motor scores of the contralateral arm or leg remained as $\leq 2$. Patients who met the following criteria were selected as group B: (1) progressive neurological signs with an increase in NIHSS score of $\geq 2$, and (2) motor score of the contralateral arm or leg increasing to $>2$ at 7 days. We compared baseline characteristics, brain images, initial NIHSS score, and NIHSS score at 7 days from symptom onset between groups A and B. Primary outcomes were set as IWI and CWI positivity rate, and secondary outcomes were set as collateral flow (anterior communicating, posterior communicating arteries) positivity rate, MCA flow positivity rate, infarctions

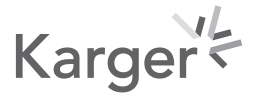


Table 1. Baseline characteristics of the study groups

\begin{tabular}{lccc}
\hline Baseline characteristics & $\begin{array}{l}\text { Group A } \\
(n=8)\end{array}$ & $\begin{array}{l}\text { Group B } \\
(n=29)\end{array}$ & $p$ \\
\hline Age (mean \pm SD), years & $76.5 \pm 10.94$ & $80.13 \pm 9.82$ & 0.590 \\
Male, $n$ (\%) & $4(50.0)$ & $11(37.9)$ & 0.830 \\
Initial NIHSS score (median, IQR) & $5(3.25)$ & $17(12.00)$ & 0.000 \\
$0-5, n$ (\%) & $4(50.0)$ & $1(3.4)$ & 0.005 \\
6-10, $n$ (\%) & $2(25.0)$ & $4(13.8)$ & 0.600 \\
$>10, n(\%)$ & $2(25.0)$ & $24(82.8)$ & 0.004 \\
Risk factors, $n$ (\%) & $4(50.0)$ & $21(72.4)$ & 0.440 \\
Hypertension & $1(12.5)$ & $6(20.7)$ & 1.000 \\
Diabetes mellitus & $2(25.0)$ & $8(27.6)$ & 1.000 \\
Dyslipidemia & $1(12.5)$ & $3(10.3)$ & 1.000 \\
Previous stroke & $3(37.5)$ & $5(17.2)$ & 0.330 \\
Smoking & $1(12.5)$ & $0(0.0)$ & 0.220 \\
Contralateral ICAD (>50\%) & $0(0.0)$ & $4(13.8)$ & 0.560 \\
IV-tPA, $n$ (\%) & $3(1)$ & $3(2)$ & 0.970 \\
Onset to imaging time (median, IQR), h & & & \\
\hline
\end{tabular}

Baseline clinical risk factors did not differ between the two groups. The initial NIHSS scores of group A indicated more mild cases than in group B. NIHSS, National Institutes of Health Stroke Scale; ICAD, internal carotid artery disease; IV-tPA, intravenous tissue plasminogen activator.

of the perforating area according to initial MRI, and deteriorated area at 7 days from symptom onset.

Categorical variables were analyzed using the Fisher exact test. Continuous variables were analyzed using the unpaired $t$ test or Mann-Whitney U test, as appropriate. Statistical significance was set at $p<0.05$. All statistical analyses were performed using EZR software (Saitama Medical Center, Jichi Medical University, Saitama, Japan) [30].

\section{Results}

\section{Clinical Information}

A total of 1,531 patients had AIS in the anterior circulation during the study period. Among them, 49 patients had symptomatic ICA occlusion with a past history of atrial fibrillation. A total of 12 of them were excluded because of endovascular treatment $(n=7)$ and incomplete MRI evaluation ( $n=5)$. Thus, 37 patients (15 men, 22 women) were finally included. Mean patient age was 79.4 years $(S D=10.06)$ and 8 patients had maintenance or improvement of their total NIHSS score and maintenance of the NIHSS motor score of the contralateral arm or leg at $\leq 2$ at 7 days from symptom onset.

Table 1 shows the general characteristics of the subjects according to their NIHSS score at 7 days from symptom onset. Age, sex, and baseline clinical risk factors did not differ between the two groups. Initial NIHSS scores indicated more mild cases in group A than in group B. There were no statistically significant differences in intravenous tissue plasminogen activator utilization rates between groups $\mathrm{A}$ and $\mathrm{B}$.

\section{Primary Outcome Measurements}

There were 3 IWI-positive cases (37.5\%) in group A and 24 IWI-positive cases in group B (82.8\%). Group A thus had a significantly lower IWI positivity rate than group B $(p=0.02)$,

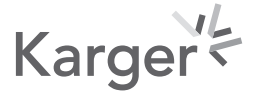


Table 2. Outcome data of the study groups

\begin{tabular}{lccc}
\hline Outcomes & $\begin{array}{l}\text { Group A } \\
(n=8)\end{array}$ & $\begin{array}{l}\text { Group B } \\
(n=29)\end{array}$ & $p$ \\
\hline Initial MRI & & & \\
DWI-ASPECTS (median, IQR) & $9(8)$ & $4(3)$ & 0.000 \\
Acom A flow positivity, $n(\%)$ & $8(100.0)$ & $16(55.2)$ & 0.030 \\
Pcom A flow positivity, $n(\%)$ & $6(75.0)$ & $15(51.7)$ & 0.420 \\
MCA flow positivity, $n$ (\%) & $5(62.5)$ & $4(13.8)$ & 0.010 \\
Perforating area infarction, $n$ (\%) & $2(25.0)$ & $25(86.2)$ & 0.002 \\
Pial area infarction, $n$ (\%) & $7(87.5)$ & $27(93.1)$ & 0.240 \\
IWI, $n$ (\%) & $3(37.5)$ & $24(82.8)$ & 0.020 \\
CWI, $n(\%)$ & $3(37.5)$ & $27(93.1)$ & 0.002 \\
IWI + CWI, $n$ (\%) & $1(12.5)$ & $20(69.0)$ & 0.012 \\
NIHSS score at 7 days (median, IQR) & $4.5(0.75)$ & $21(17)$ & 0.000 \\
0-5, $n$ (\%) & $6(75.0)$ & $0(0.0)$ & 0.000 \\
6-10, $n$ (\%) & $1(12.5)$ & $1(3.4)$ & 0.390 \\
>10, $n$ (\%) & $1(12.5)$ & $28(96.6)$ & 6.040 \\
MR or CT images at 7 days & & & \\
DWI, CT-ASPECTS (median, IQR) & $8(7)$ & $2(0)$ & 0.000 \\
Deteriorated regions & & & \\
Perforating area, $n$ (\%) & $2(25.0)$ & $20(69.0)$ & 0.040 \\
Pial area, $n$ (\%) & $3(37.5)$ & $12(41.4)$ & 1.000 \\
\hline
\end{tabular}

Group A had significantly lower IWI and CWI positivity rates than group B $(p=0.02,0.002)$, as indicated using Fisher's exact test. In group A, there were significantly higher positivity rates of Acom artery flow and MCA flow, and lower rates of infarctions in the perforating area at initial MRI ( $p=0.03,0.01$, and 0.002) according to Fisher's exact test. MRI, magnetic resonance imaging; DWI, diffusion-weighted images; ASPECTS, Alberta Stroke Program Early CT Score; Acom A, anterior communicating artery; Pcom A, posterior communicating artery; MCA, middle cerebral artery; IWI, internal watershed infarction; CWI, cortical watershed infarction; NIHSS, National Institutes of Health Stroke Scale; CT, computed tomography.

as assessed using Fisher's exact test. There were 3 (37.5\%) CWI-positive cases in group A and 27 (93.1\%) in group B. Group A had a statistically significantly lower CWI positivity rate than group B ( $p=0.002)$, as assessed using Fisher's exact test (Table 2).

\section{Secondary Outcome Measurements}

Group A had significantly higher rates of anterior communicating artery flow positivity and MCA flow positivity, and lower rates of infarctions of the perforating area in initial MRI ( $p=0.03,0.01$, and 0.002 , respectively), as assessed using Fisher's exact test. The rate of deteriorated infarctions in the perforating area at 7 days from symptom onset was also statistically significantly lower in group A than group B $(p=0.04)$, as seen using Fisher's exact test (Table 2).

\section{Discussion}

Both IWI and CWI positivity rates were higher in patients whose NIHSS scores worsened by $>2$ at 7 days from symptom onset as compared to patients with a good clinical course (whose NIHSS score did not change or improved at 7 days from symptom onset). These outcomes supported our hypothesis. In our study, lesions in the perforating area showed statistically greater deterioration in patients whose NIHSS scores worsened by $>2$ at 7 days 
from symptom onset. This suggests a correlation between WSI and lesions of the perforating area.

Many reports have implicated hemodynamic impairment as a determinant of IWI, particularly in cases with small, deep infarcts in the centrum semiovale (rosary-like watershed infarcts) $[16,19,21,22,24]$. Several characteristics of IWIs have been pointed out, such as vulnerability of the vasculature of the centrum semiovale [23, 25], their association with severe systemic hypotension $[21,23,25]$, significant component of an incompletely infarcted brain on pathological studies [31], and impaired cerebral vasoreactivity associated with oxygen metabolism failure [16, 32].

Lenticulostriate arteries (LSAs) are among the largest perforating arteries in the brain. These vessels originate from the MCA and range from 2 to 12 in number [33]. ICA occlusion leads to a decrease in anterograde blood flow in the LSAs. This decrease in blood flow is compensated by retrograde flow from the ICA and the pial branch of the posterior cerebral artery. Since the LSAs are mostly located distally, perfusion pressure is likely to be the lowest for these vessels. Therefore, we considered that hypoperfusion is a significant underlying mechanism of infarction in the territory supplied by the LSA, which is similar to the mechanism underlying IWI.

Conversely, CWI is regarded as being induced by a microembolic mechanism [26, 29] because of a lower level of impairment of cerebral vasoreactivity $[27,28]$ and oxygen metabolism $[16,20]$. There is reportedly a positive correlation between cerebral perfusion and the number of LSAs [34], with occlusion of an individual LSA usually resulting in large ganglionic infarcts [35]. If microembolism of an LSA occurs, blood flow to the basal ganglia decreases encouraging hypoperfusion in the LSA. Therefore, we believe that microembolism of the LSAs results in deterioration of hypoperfusion in the LSA, resulting in a large ganglionic infarct.

The results of this study are subject to some limitations. First, since this was a retrospective study with a small sample size, further studies with a larger sample size are required to confirm our results. Second, we evaluated the initial MRI within $24 \mathrm{~h}$ of symptom onset (median time of both groups A and group B: $3 \mathrm{~h}$ ). There is no evidence suggesting the best timing for initial assessment of WSI.

\section{Conclusion}

In patients with mild symptoms who are found to have symptomatic ICA occlusion, presence of cortical and internal watershed infarcts might be key signs for predicting neurological deterioration after hospitalization.

\section{Statement of Ethics}

The Ethics Committee of Seirei Mikatahara General Hospital approved the protocol for this study, which was performed in accordance with the principles of the Declaration of Helsinki.

\section{Conflict of Interest Statement}

No company had influence on or knowledge of the results of this study.

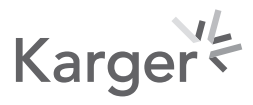




\section{Cerebrovascular Diseases Extra}

\author{
Funding Sources
}

The authors received no funding for this work.

\title{
Author Contributions
}

Conception and design: Y. Amano, A. Fujimoto, S. Akamine. Acquisition of data: Y. Amano. Statistical analysis: Y. Amano. Analysis and interpretation of data: H. Sano, H. Kenmochi. Reviewed submitted version of manuscript: A. Fujimoto, H. Sato. Study supervision: S. Akamine.

\section{References}

1 Berkhemer OA, Kamalian S, González RG, Majoie CB, Yoo AJ. Imaging Biomarkers for Intra-arterial Stroke Therapy. Cardiovasc Eng Technol. 2013 Dec;4(4):339-51.

2 Berkhemer OA, Fransen PS, Beumer D, van den Berg LA, Lingsma HF, Yoo AJ, et al.; MR CLEAN Investigators. A randomized trial of intraarterial treatment for acute ischemic stroke. N Engl J Med. 2015 Jan;372(1):11-20.

3 Goyal M, Demchuk AM, Menon BK, Eesa M, Rempel JL, Thornton J, et al.; ESCAPE Trial Investigators. Randomized assessment of rapid endovascular treatment of ischemic stroke. N Engl J Med. 2015 Mar;372(11):1019-30.

4 Jovin TG, Chamorro A, Cobo E, de Miquel MA, Molina CA, Rovira A, et al.; REVASCAT Trial Investigators. Thrombectomy within 8 hours after symptom onset in ischemic stroke. N Engl J Med. 2015 Jun;372(24):2296-306.

5 Saver JL, Goyal M, Bonafe A, Diener HC, Levy EI, Pereira VM, et al.; SWIFT PRIME Investigators. Stent-retriever thrombectomy after intravenous t-PA vs. t-PA alone in stroke. N Engl J Med. 2015 Jun;372(24):2285-95.

6 Bracard S, Ducrocq X, Mas JL, Soudant M, Oppenheim C, Moulin T, et al.; THRACE investigators. Mechanical thrombectomy after intravenous alteplase versus alteplase alone after stroke (THRACE): a randomised controlled trial. Lancet Neurol. 2016 Oct;15(11):1138-47.

7 Muir KW, Ford GA, Messow CM, Ford I, Murray A, Clifton A, et al.; PISTE Investigators. Endovascular therapy for acute ischaemic stroke: the Pragmatic Ischaemic Stroke Thrombectomy Evaluation (PISTE) randomised, controlled trial. J Neurol Neurosurg Psychiatry. 2017 Jan;88(1):38-44.

8 Albers GW, Marks MP, Kemp S, Christensen S, Tsai JP, Ortega-Gutierrez S, et al.; DEFUSE 3 Investigators. Thrombectomy for Stroke at 6 to 16 Hours with Selection by Perfusion Imaging. N Engl J Med. 2018 Feb; 378(8):708-18.

9 Nogueira RG, Jadhav AP, Haussen DC, Bonafe A, Budzik RF, Bhuva P, et al.; DAWN Trial Investigators. Thrombectomy 6 to 24 Hours after Stroke with a Mismatch between Deficit and Infarct. N Engl J Med. 2018 Jan; 378(1):11-21.

10 Powers WJ, Rabinstein AA, Ackerson T, Adeoye OM, Bambakidis NC, Becker K, et al.; American Heart Association Stroke Council. 2018 Guidelines for the Early Management of Patients With Acute Ischemic Stroke: A Guideline for Healthcare Professionals From the American Heart Association/American Stroke Association. Stroke. 2018 Mar; 49(3):e46-110.

11 Mokin M, Masud MW, Dumont TM, Ahmad G, Kass-Hout T, Snyder KV, et al. Outcomes in patients with acute ischemic stroke from proximal intracranial vessel occlusion and NIHSS score below 8. J Neurointerv Surg. 2014 Jul;6(6):413-7.

12 Heldner MR, Jung S, Zubler C, Mordasini P, Weck A, Mono ML, et al. Outcome of patients with occlusions of the internal carotid artery or the main stem of the middle cerebral artery with NIHSS score of less than 5: comparison between thrombolysed and non-thrombolysed patients. J Neurol Neurosurg Psychiatry. 2015 Jul; 86(7):755-60.

13 Paciaroni M, Caso V, Venti M, Milia P, Kappelle LJ, Silvestrelli G, et al. Outcome in patients with stroke associated with internal carotid artery occlusion. Cerebrovasc Dis. 2005;20(2):108-13.

14 Klijn CJ, Kappelle LJ, Tulleken CA, van Gijn J. Symptomatic carotid artery occlusion. A reappraisal of hemodynamic factors. Stroke. 1997 Oct;28(10):2084-93.

15 Dyken ML. Intracranial 'steal' in complete occlusion of the internal carotid artery. Variations in response to 5 per cent carbon dioxide and 100 per cent oxygen. Eur Neurol. 1971-1972;6(1):301-5.

16 Derdeyn CP, Khosla A, Videen TO, Fritsch SM, Carpenter DL, Grubb RL Jr, et al. Severe hemodynamic impairment and border zone-region infarction. Radiology. 2001 Jul;220(1):195-201.

17 Caplan LR, Hennerici M. Impaired clearance of emboli (washout) is an important link between hypoperfusion, embolism, and ischemic stroke. Arch Neurol. 1998 Nov;55(11):1475-82.

18 Van den Bergh R. Centrifugal elements in the vascular pattern of the deep intracerebral blood supply. Angiology. 1969 Feb;20(2):88-94.

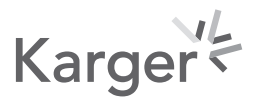


19 Provinciali L, Ceravolo MG, Minciotti P. A transcranial Doppler study of vasomotor reactivity in symptomatic carotid occlusion. Cerebrovasc Dis. 1993;3(1):27-32.

20 Arakawa S, Minematsu K, Hirano T, Tanaka Y, Hasegawa Y, Hayashida K, et al. Topographic distribution of misery perfusion in relation to internal and superficial borderzones. AJNR Am J Neuroradiol. 2003 Mar;24(3): 427-35.

21 Brierley JB, Excell BJ. The effects of profound systemic hypotension upon the brain of M. rhesus: physiological and pathological observations. Brain. 1966 Jun;89(2):269-98.

22 Ringelstein EB, Sievers C, Ecker S, Schneider PA, Otis SM. Noninvasive assessment of CO2-induced cerebral vasomotor response in normal individuals and patients with internal carotid artery occlusions. Stroke. 1988 Aug;19(8):963-9.

23 Moody DM, Bell MA, Challa VR. Features of the cerebral vascular pattern that predict vulnerability to perfusion or oxygenation deficiency: an anatomic study. AJNR Am J Neuroradiol. 1990 May;11(3):431-9.

24 Weiller C, Ringelstein EB, Reiche W, Buell U. Clinical and hemodynamic aspects of low-flow infarcts. Stroke. 1991 Sep;22(9):1117-23.

25 Bladin CF, Chambers BR. Clinical features, pathogenesis, and computed tomographic characteristics of internal watershed infarction. Stroke. 1993 Dec;24(12):1925-32.

26 Yong SW, Bang OY, Lee PH, Li WY. Internal and cortical border-zone infarction: clinical and diffusion-weighted imaging features. Stroke. 2006 Mar;37(3):841-6.

27 Moriwaki H, Matsumoto M, Hashikawa K, Oku N, Ishida M, Seike Y, et al. Hemodynamic aspect of cerebral watershed infarction: assessment of perfusion reserve using iodine-123-iodoamphetamine SPECT. J Nucl Med. 1997 Oct;38(10):1556-62.

28 Bisschops RH, Klijn CJ, Kappelle LJ, van Huffelen AC, van der Grond J. Association between impaired carbon dioxide reactivity and ischemic lesions in arterial border zone territories in patients with unilateral internal carotid artery occlusion. Arch Neurol. 2003 Feb;60(2):229-33.

29 Sorgun MH, Rzayev S, Yilmaz V, Isıkay CT. Etiologic subtypes of watershed infarcts. J Stroke Cerebrovasc Dis. 2015 Nov;24(11):2478-83.

30 Kanda Y. Investigation of the freely available easy-to-use software 'EZR' for medical statistics. Bone Marrow Transplant. 2013 Mar;48(3):452-8.

31 Lammie GA, Wardlaw JM. Small centrum ovale infarcts-a pathological study. Cerebrovasc Dis. 1999 Mar-Apr; $9(2): 82-90$.

32 Momjian-Mayor I, Baron JC. The pathophysiology of watershed infarction in internal carotid artery disease: review of cerebral perfusion studies. Stroke. 2005 Mar;36(3):567-77.

33 Djulejić V, Marinković S, Maliković A, Jovanović I, Djordjević D, Cetković M, et al. Morphometric analysis, region of supply and microanatomy of the lenticulostriate arteries and their clinical significance. J Clin Neurosci. 2012 Oct;19(10):1416-21.

34 Chen YC, Wei XE, Lu J, Qiao RH, Shen XF, Li YH. Correlation between the number of lenticulostriate arteries and imaging of cerebral small vessel disease. Front Neurol. 2019 Aug;10:882.

35 Marinković S, Gibo H, Milisavljević M, Ćetković M. Anatomic and clinical correlations of the lenticulostriate arteries. Clin Anat. 2001 May;14(3):190-5. 